\title{
Association between serum uric acid level and carotid atherosclerosis and metabolic syndrome in patients with type 2 diabetes mellitus
}

Wei Li ( $D$ libuwei2011@163.com )

Zhengzhou University First Affiliated Hospital https://orcid.org/0000-0002-4073-4397

\section{Yan Wang}

Zhengzhou University First Affiliated Hospital

\section{Shengrong Ouyang}

Capital Institute of Pediatrics

Mengdi Li

Zhengzhou University First Affiliated Hospital

Rui Liu

Zhengzhou University First Affiliated Hospital

Yuqi Zhang

Zhengzhou University First Affiliated Hospital

\section{Xiaojun Liu}

Zhengzhou University First Affiliated Hospital

\section{Tianfang Li}

Zhengzhou University First Affiliated Hospital

\section{Shengyun Liu}

Zhengzhou University First Affiliated Hospital

\section{Research article}

Keywords: Uric acid, Metabolic syndrome, Carotid atherosclerosis, Intima-media thickness, Type 2 diabetes mellitus

Posted Date: November 10th, 2021

DOI: https://doi.org/10.21203/rs.3.rs-961621/v2

License: (c) (1) This work is licensed under a Creative Commons Attribution 4.0 International License. Read Full License 


\section{Abstract \\ Background}

Serum uric acid (SUA) is associated with many cardiovascular risk factors, such as metabolic syndrome (MetS) and subclinical atherosclerosis. However, the relationship of SUA with carotid atherosclerosis remains controversial. We aimed to investigate whether elevated SUA levels are associated with a high risk of carotid atherosclerosis and MetS in patients with type 2 diabetes mellitus (T2DM).

\section{Methods}

This cross-sectional study was performed with a sample of 1,947 hospitalized patients with T2DM. Carotid intima-media thickness (CIMT) and carotid artery plaques (CAP) were measured via Doppler ultrasound.

\section{Results}

Uric acid levels were negatively associated with HbA1C, eGFR, and HDL-C (all $\mathrm{P}<0.001)$ and positively associated with WBC, BMI, ACR, creatinine, total cholesterol, triglycerides, LDL-C, systolic blood pressure, and diastolic blood pressure (all $\mathrm{P}<0.001$ ). After adjusting for multiple potential confounders, the risks were substantially higher for MetS in the highest quartile of SUA levels (odds ratio: 2.91, 95\% confidence interval: $1.54-5.51, P=0.003$ for trend) than in the lowest quartile of SUA levels. Furthermore, a significant increase was observed in the prevalence of overweight/obesity, hypertension, and dyslipidemia across the SUA quartiles independent of confounders. However, no significant association was found between SUA quartile with the presence of carotid atherosclerosis.

\section{Conclusions}

In patients with T2DM, SUA levels were closely associated with MetS and its components but not with carotid atherosclerosis.

\section{Background}

Accumulating epidemiological and clinical evidence demonstrated that serum uric acid (SUA) levels are strongly associated with many cardiovascular risk factors, such as obesity, hypertension, hyperlipidemia, diabetes, metabolic syndrome (MetS), and subclinical atherosclerosis[1-3]. Among these risk factors, the associations of SUA with atherosclerosis and MetS have been observed in several studies in both general and type 2 diabetic mellitus (T2DM) populations[4, 5]. 
Uric acid is the end metabolic product of purine in humans. Hyperuricemia can lead to various diseases, and it is most notably involved in the pathogenesis of gouty arthritis[3]. Previous studies have suggested that hyperuricemia is a risk factor for cardiovascular disease (CVD) in the general population[6, 7]. Ultrasound of the carotid artery to identify carotid intima-media thickness (CIMT) and carotid artery plaques (CAP) can predict the risk of CVD. As a surrogate of atherosclerosis diseases, CAP accounts for approximately a fifth of the risk of stroke and coronary artery diseases[8, 9]. However, the associations between SUA concentration and carotid atherosclerosis, as reflected by CIMT and CAP, have previously been studied but produced conflicting results. A population-based cross-sectional survey demonstrated that SUA level is associated with MetS and an independent risk factor for carotid atherosclerosis in patients with T2DM[4]. Another study showed that SUA levels are closely associated with hypertension and MetS but not with atherosclerosis in people with diabetes[10]. Some authors considered that the role of uric acid in atherosclerosis might be attributed to other cardiovascular risk factors, such as hypertension, obesity, MetS, and chronic kidney disease[11, 12].

Although previous studies have showed that the independence of relationship between SUA and MetS and atherosclerosis. However, few studies have examined the relationship between SUA and components of MetS, as well as the association between SUA and carotid atherosclerosis in patients with T2DM. Therefore, this study aimed to investigate the association between SUA level and MetS and carotid atherosclerosis in T2DM populations.

\section{Methods}

Study subjects

This cross-sectional study evaluated the prevalence of MetS and carotid atherosclerosis in patients with T2DM aged over 18 who were hospitalized at the First Affiliated Hospital of Zhengzhou University from January 2018 to December 2020. Patients who were taking any drug that might interfere with uric acid metabolism, such as allopurinol and furosemide, were excluded. Patients who did not undergo carotid ultrasound examination and without complete clinical and SUA data were also excluded. In total, 1,947 patients, including 1,335 males, were included in the final analyses. All patients underwent an interview and provided a history of hypertension, CVD, duration of diabetes, use of lipid-lowering drugs and antihypertensive agents, alcohol consumption, and smoking habits. Body mass index (BMI) was calculated by body weight $(\mathrm{kg})$ divided by height squared $\left(\mathrm{m}^{2}\right)$. Blood pressure was measured by using an automatic blood-pressure meter after the participants sat for at least $10 \mathrm{~min}$. The average of three measurements was recorded for further analysis. This study was approved by the Institution Review Board of the First Affiliated Hospital of Zhengzhou University.

\section{Laboratory measurements}

The patients were asked to fast overnight, and then blood samples were obtained for further analysis. Total cholesterol (TC), high-density lipoprotein cholesterol (HDL-C), low-density lipoprotein cholesterol 
(LDL-C), triglycerides (TG), fasting blood glucose (FBG), insulin, SUA, creatinine, white blood cells (WBC), and $\mathrm{C}$-reactive protein (CRP) were measured. $\mathrm{HbA} 1 \mathrm{c}$ level was measured via high-performance liquid chromatography. A sterile, random-spot urine sample was used to measure the albumin/creatinine ratio (ACR). The estimated glomerular filtration rate (eGFR) was calculated using the simplified Modification of Diet in Renal Disease formula: eGFR $=186.3 \times(\text { serum creatinine })^{-1.154} \times(\text { age })^{-0.203} \times(0.742$ if female $)$.

Assessment of CAP and CIMT

Carotid ultrasonography was performed using a color Doppler ultrasonic diagnostic instrument. Trained and certified sonographers conducted the examination. CIMT was determined at the point approximately $1.5 \mathrm{~cm}$ away from the distal part of the bifurcation of common carotid artery. CIMT was calculated as the mean of the intima-media thicknesses of the left and right common carotid arteries. CAP was defined as a focal region with a thickness of $\geq 1.5 \mathrm{~mm}$ as measured from the media adventitia interface to the lumen-intima interface or as the presence of focal wall thickening that was at least $50 \%$ greater than that of the surrounding vessel wall.

Definition of MetS

MetS was defined on the basis of the updated National Cholesterol Education Program Adult Treatment Panel III criteria for Asian-Americans as presenting at least three of the following components: 1) waist circumference $90 \mathrm{~cm}$ or greater in men or $80 \mathrm{~cm}$ or greater in women; 2) TG $1.7 \mathrm{mmol} / \mathrm{L}$ or greater; 3) $\mathrm{HDL}-\mathrm{C}$ less than $1.03 \mathrm{mmol} / \mathrm{L}$ in men or less than $1.30 \mathrm{mmol} / \mathrm{L}$ in women; 4) blood pressure 130/85 $\mathrm{mmHg}$ or greater or current use of antihypertensive medications; or 5) fasting plasma glucose 5.6 $\mathrm{mmol} / \mathrm{L}$ or greater or previously diagnosed with T2DM or on oral antidiabetic agents or insulin[13].

\section{Statistical analysis}

Normally distributed data were expressed as means $\pm S D$, whereas variables with a skewed distribution were reported as median (interquartile range, IQR). Categorical variables were represented by frequency and percentage. Kruskal-Wallis test was used to analyze groups differences for continuous variables, and Chi-square test was used for categorical variables. Spearman correlation coefficients between SUA and metabolic features were calculated by partial correlation analysis on ranks. Multivariate logistic regression models were used to estimate the odds ratios (ORs) for CAP and MetS according to SUA quartiles. Potential confounding variables, including age, gender, smoking, alcohol drinking, duration of diabetes, self-reported CVD, eGFR, FBG, HbA1C, CRP, and BMI, were controlled in the regression models. All statistical analyses were performed using SPSS version 26.0 (SPSS, Chicago, IL, USA). P 0.05 was considered statistically significant.

\section{Results}

Characteristics of the participants according to SUA quartiles 
We identified 1,947 patients with T2DM with a mean age of $49.6 \pm 11.9$ years. The population studied herein was stratified into quartiles according to SUA levels. The baseline demographic and medical characteristics for SUA quartiles are provided in Table 1. The cut-off SUA values for Q1, Q2, Q3, and Q4 were $<242,242-293,293-353$, and $\geq 353 \mu \mathrm{mol} / \mathrm{L}$, respectively. When analyzed by quartiles of SUA levels, the patients with higher uric acid levels were more likely to be male, smokers, drinker, and younger (all $\mathrm{P}<$ 0.001). With respect to metabolic parameters, the patients in the higher uric acid quartiles exhibited higher levels of systolic blood pressure (SBP) and diastolic blood pressure (DBP), BMI, CRP, creatinine, insulin, ACR, TG, and TC than those in the lower uric acid quartiles (all $P<0.05$ ). By contrast, the patients with higher uric acid levels displayed shorter duration of diabetes and lower levels of HbA1C and HDL-C than those with lower uric acid levels (all $P<0.05$ ). However, no difference in CIMT and CAP was observed between the SUA quartile groups.

Correlation between SUA and other parameters

Partial correlation analysis revealed close correlation between SUA levels and BMI, HbA1C, ACR, creatinine, eGFR, SBP, DBP, TC, TG, and HDL-C among various metabolic features after adjusting for age, gender, and duration of diabetes (Table 2). Remarkably, SUA levels gradually increased with increasing number of MetS components. The mean values of SUA concentrations significantly increased for those with one, two, three, four, and five components of MetS; the mean values were $244.3 \pm 62.7,266.9 \pm 75.5$, $298.7 \pm 92.5,322.2 \pm 94.3$, and $337.7 \pm 98.2 \mu \mathrm{mol} / \mathrm{L}$, respectively $(P<0.001$, Fig. 1a). Furthermore, the prevalence of MetS was higher with increasing SUA quartiles; $57.10 \%, 72.80 \%, 80.20 \%$, and $87.90 \%$ for Q1, Q2, Q3, and Q4, respectively ( $<0.001$ for trend, Fig. 1b).

Comparison of MetS and CAP between the SUA quartile groups

As presented in Table 3, the OR for MetS was higher with increasing SUA quartiles after adjusting for age and gender (OR: 5.132, 95\% Cl: 3.63-7.25, $\mathrm{P}<0.001$ for trend). In the highest uric acid quartile, the OR was 2.91 (95\% Cl: 1.54-5.51, P = 0.003 for trend) for MetS after further adjusting for alcohol drinking, smoking, duration of diabetes, self-reported CVD, BMI, CRP, HbA1C, FBG, eGFR, TC, and LDL-C. A comparison of CAP between the SUA quartile groups after adjusting for multiple potential confounders is given in Table 3. However, no significant differences were observed in the prevalence of CAP across the four groups.

Association between SUA quartiles and other MetS components

The associations of the SUA quartiles with different MetS components in all patients with T2DM are summarized in Table 4. After controlling for multiple confounding factors, the SUA quartiles were found to be independently associated with increased prevalence of hypertension in T2DM populations. The OR for hypertension increased with increasing SUA quartiles. In the highest SUA quartile, the OR was 2.13 (95\% Cl: 1.34-3.38, P = 0.015) for hypertension. Similarly, in the highest SUA quartile, the OR was 3.45 (95\% Cl: 2.25-5.28, P < 0.001) for overweight/obesity (BMI $\geq 25 \mathrm{~kg} / \mathrm{m}^{2}$ ). Dyslipidemia was defined as TC $\geq 6.2 \mathrm{mmol} / \mathrm{L}$, or TG $=1.7 \mathrm{mmol} / \mathrm{L}$ or greater, or HDL-C $<1.03 \mathrm{mmol} / \mathrm{L}$ in men or $<1.30 \mathrm{mmol} / \mathrm{L}$ in 
women. In our patients with T2DM, after controlling for confounding factors, the SUA quartiles were still independently associated with increased prevalence of dyslipidemia. In the highest SUA quartile, the OR for dyslipidemia ( $T \mathrm{G} \geq 1.7 \mathrm{mmol} / \mathrm{L}$ ) was 2.71 (95\% Cl: 1.73-4.25, $\mathrm{P}<0.001)$, and the OR for dyslipidemia ( $\mathrm{TC} \geq 6.2 \mathrm{mmol} / \mathrm{L}$ ) was 2.51 (95\% Cl: 1.17-5.38, P = 0.013). However, no significant differences were found in the prevalence of dyslipidemia (HDL-C abnormality) across the four SUA quartile groups.

\section{Discussion}

SUA levels were strongly associated with the presence of MetS but not with the presence of carotid atherosclerosis in the patients with T2DM. In the highest SUA quartile, the ORs were 2.91 (95\% Cl: 1.545.51) for MetS after further adjusting for other atherosclerotic risk factors, such as age, gender, BMI, eGFR, and other lipid and glycemic parameters. However, SUA quartile was not associated with the presence of carotid artery plaque. Furthermore, the prevalence of hypertension, dyslipidemia, and overweight/obesity, substantially increased across the SUA quartiles independent of potential confounders.

Many studies have evaluated the associations between uric acid and MetS[14, 15]. Consistent with the results of previous studies[4, 10], we observed a strong relationship between SUA and MetS. The ORs were substantially higher for MetS (OR: $2.91,95 \% \mathrm{Cl} 1.54-5.51, \mathrm{P}=0.003$ for trend) in the highest SUA quartile than those in the lowest SUA quartile. We also observed that SUA concentration increased with the number of MetS components $(P<0.001$ for trend). Moreover, we found that the patients in the higher SUA quartiles had greater numbers of MetS components than the patients in the lower SUA quartiles. However, the underlying mechanisms of the association between SUA and MetS remain largely unknown. Previous studies indicated that hyperuricemia may be partially responsible for the inflammatory process in adipose tissues and vascular endothelial cells that will lead to a chronic low-grade inflammation and insulin resistance in subjects with MetS[16, 17]. A recent study showed that higher SUA levels present higher levels of CRP and increased serum levels of inflammatory cytokines, indicating that SUA may be an inductor of subclinical inflammation[18]. Consistent with this supposition, we observed that the acute phase biomarkers, including WBC and CRP levels, gradually increased with SUA quartiles $(P<0.001)$. Therefore, given that low-grade inflammation and insulin resistance are two major risks factors for MetS, uric acid-induced inflammatory pathway may play an important role in the pathogenesis of MetS. Interestingly, previous studies have demonstrated that reducing uric acid substantially improves systemic inflammation, endothelial function, and peripheral vasodilator capacity[19, 20]. Thus, SUA may be a promising candidate for risk assessment and a potential intervention target for MetS and CVD[16].

Numerous studies have demonstrated that SUA levels are independently associated with the presence of hypertension[21, 22]. A recent study suggested that uric acid has independent effects on the development of hypertension and MetS but is not an independent risk factor for atherosclerosis in patients with T2DM[10]. Our results were in agreement with these findings. The ORs for hypertension increased with increasing SUA quartiles. In the highest SUA quartile, the OR was $2.13(95 \% \mathrm{Cl}: 1.34-3.38, \mathrm{P}<0.015)$ for hypertension. Experimental studies have also suggested the potential roles of uric acid in the 
pathogenesis of hypertension. The mechanism by which uric acid causes hypertension may be due to the inhibition of the release of endothelial nitric oxide and the activation of the rennin-angiotensin system, which lead to oxidative stress, endothelial dysfunction, and smooth muscle cell proliferation, and ultimately to elevated blood pressure[23, 24].

In the past decades, several studies have assessed the relationship between SUA and the components of MetS in different selected populations. Multiple clinical and epidemiological studies have also demonstrated the strong association among SUA and obesity, hypertension, and MetS[25, 26]. A previous study reported that the dyslipidemia components of serum TC, TG, and LDL-C levels are positively associated with SUA levels, whereas serum HDL-C levels are inversely related with SUA[27]. Our analysis were consistent with these findings. A notable increase in the risk of overweight/obesity, hypertension, and dyslipidemia was observed across the SUA quartiles after adjusting for known potential confounders. However, the issue of whether hyperuricemia is a downstream result of MetS or it may play an upstream role in MetS development remains unclear. The Mendelian randomization (MR) technique enables their use as instrumental variables for testing causality by exploiting the random distribution of genetic variants[28]. An MR investigation suggested that SUA may augment the risk of MetS by increasing blood pressure and TC levels and lowering HDL-C levels but not by accumulating fat or hyperglycemia. Obesity may be a causal agent for all the components of MetS, including hyperuricemia[29]. Meanwhile, a recent study of obese adults indicated that SUA has no apparent association with hypertension, dyslipidemia, T2DM, and cardiovascular events[30]. Thus, further complementary studies on the causal relationship and the potential mechanism between SUA and components of MetS are warranted.

However, the results regarding the associations of SUA with atherosclerosis and CVD from different studies remains controversial[4,31]. Several clinical studies have reported that elevated SUA levels are independent predictors of atherosclerosis, CVD, and mortality in different populations[5]. However, other epidemiological studies have failed to confirm such associations and argued that these relationship are not causal but rather a result of a coexistence with other cardiovascular risk factors, such as obesity, MetS, and chronic kidney disease[10, 11]. A recent study indicated that SUA appears to be strongly correlated with CIMT but not with the prevalence of carotid plaques or aortic stiffness[23]. The present study did not observe any association between SUA and CIMT and carotid atherosclerotic plaques in T2DM populations despite adjusting for all known confounders. Thus, these results demonstrated that SUA may not be a risk factor for CAP and that the association between SUA and carotid atherosclerosis is not truly independent. Additionally, the methodological differences and the different characteristics of study populations might account for the discrepancies reported in the literature and by the present study. Therefore, given that the SUA levels were strongly associated with MetS and its components, SUA may play an indirect role in the pathogenesis of atherosclerosis via other CVD factors, such as obesity, hypertension, dyslipidemia, and MetS in some selected populations.

Owing to the cross-sectional nature of this study, it has several limitations. The mechanisms underlying these associations remain to be explored. The study findings are inherently limited in the ability to 
eliminate causal effect relationships between SUA and MetS. The participants of the present study were Chinese patients with T2DM. Therefore, the present results might not be representative of the general population. Moreover, many cardiovascular risk factors, such as glucose and lipid metabolic disorders, can accumulate in patients with T2DM and might affect the role of SUA in the development of carotid atherosclerosis and CVD.

\section{Conclusion}

In summary, the findings of this study strongly suggested that SUA has independent association with the prevalence of hypertension, obesity, dyslipidemia and MetS but not with carotid atherosclerosis in T2DM populations. Our findings demonstrated that the role of uric acid in atherosclerosis might be attributed to other cardiovascular risk factors, such as MetS and its components. Prospective studies are required to clarify further the causal associations of SUA with MetS and carotid atherosclerosis in patients with T2DM.

\section{Abbreviations}

ACR

Albumin/creatinine ratio

BMI

Body mass index

CAP

Carotid artery plaques

$\mathrm{Cl}$

Confidence interval

CIMT

Carotid intima-media thickness

CVD

Cardiovascular disease

DBP

Diastolic blood pressure

eGFR

Estimated glomerular filtration rate

FBG

Fasting plasma glucose

$\mathrm{HbA1c}$

Glycosylated hemoglobin

HDL-C

High-density lipoprotein cholesterol

LDL-C 
Low-density lipoprotein cholesterol

MetS

metabolic syndrome

OR

Odds ratio

SBP

Systolic blood pressure

TC

Total cholesterol

TG

\section{Triglyceride}

T2DM

Type 2 diabetes mellitus

SUA

Serum uric acid.

\section{Declarations}

\section{Acknowledgements}

We would like to thank the participants in this study.

\section{Authors' contributions}

WL, YW, TFL, XJL, and SYL contributed to the conception and design of the study. WL, YW, SYL, YQZ, $M D L$, and $R L$ recruited the subjects and supervised the study. $W L, Y W$ and $S R O$ analyzed the data. WL and SYL wrote the initial draft of the paper. WL, YW, TFL, XJL, and SYL contributed to the writing, reviewing, and revising of the manuscript. All authors read and approved the final manuscript.

\section{Funding}

WL is funded by the National Natural Science Foundation of China (82000831).

\section{Availability of data}

The datasets in the current study are available from the corresponding author upon reasonable request.

\section{Ethics approval and consent to participate}

The study protocol was approved by the Ethical Committee of the First Affiliated Hospital of Zhengzhou University, and the requirement for informed consent was exempted. 
The corresponding author and all the authors have read and approved the final submitted manuscript, and agreed to conditions noted on the Author Disclosure Form. No portion of the work has been or is currently under consideration for publication elsewhere. No portion of the manuscript has been published.

\section{Competing interests}

The authors declare that they have no competing interests.

\section{References}

1. Lim DH, Lee Y, Park GM, et al. Serum uric acid level and subclinical coronary atherosclerosis in asymptomatic individuals: An observational cohort study. Atherosclerosis 2019;288:112-7.

2. Li X, Meng X, He Y, et al. Genetically determined serum urate levels and cardiovascular and other diseases in UK Biobank cohort: A phenome-wide mendelian randomization study. PLoS Med 2019;16(10):e1002937.

3. Dehlin M, Jacobsson L, Roddy E. Global epidemiology of gout: prevalence, incidence, treatment patterns and risk factors. Nat Rev Rheumatol 2020;16(7):380-90.

4. Li Q, Yang Z, Lu B, et al. Serum uric acid level and its association with metabolic syndrome and carotid atherosclerosis in patients with type 2 diabetes. Cardiovasc Diabetol 2011;10:72.

5. Wu SS, Kor CT, Chen TY, et al. Relationships between Serum Uric Acid, Malondialdehyde Levels, and Carotid Intima-Media Thickness in the Patients with Metabolic Syndrome. Oxid Med Cell Longev 2019;2019:6859757.

6. Johnson RJ, Bakris GL, Borghi C, et al. Hyperuricemia, Acute and Chronic Kidney Disease, Hypertension, and Cardiovascular Disease: Report of a Scientific Workshop Organized by the National Kidney Foundation. Am J Kidney Dis 2018;71(6):851-65.

7. Mortada I. Hyperuricemia, Type 2 Diabetes Mellitus, and Hypertension: an Emerging Association. Curr Hypertens Rep 2017;19(9):69.

8. Naqvi TZ, Lee MS. Carotid intima-media thickness and plaque in cardiovascular risk assessment. JACC Cardiovasc Imaging 2014;7(10):1025-38.

9. Kopczak A, Schindler A, Bayer-Karpinska A, et al. Complicated Carotid Artery Plaques as a Cause of Cryptogenic Stroke. J Am Coll Cardiol 2020;76(19):2212-22.

10. Li LX, Dong XH, Li MF, et al. Serum uric acid levels are associated with hypertension and metabolic syndrome but not atherosclerosis in Chinese inpatients with type 2 diabetes. $J$ Hypertens 2015;33(3):482-90; discussion 90.

11. Li LX, Wang AP, Zhang $R$, et al. Decreased urine uric acid excretion is an independent risk factor for chronic kidney disease but not for carotid atherosclerosis in hospital-based patients with type 2 diabetes: a cross-sectional study. Cardiovasc Diabetol 2015;14:36.

12. Chen MY, Zhao CC, Li TT, et al. Serum uric acid levels are associated with obesity but not cardiocerebrovascular events in Chinese inpatients with type 2 diabetes. Sci Rep 2017;7:40009. 
13. Grundy SM, Cleeman JI, Daniels SR, et al. Diagnosis and management of the metabolic syndrome: an American Heart Association/National Heart, Lung, and Blood Institute Scientific Statement. Circulation 2005;112(17):2735-52.

14. Tani S, Matsuo R, Imatake K, et al. The serum uric acid level in females may be a better indicator of metabolic syndrome and its components than in males in a Japanese population. $J$ Cardiol 2020;76(1):100-8.

15. Kawamoto R, Ninomiya D, Kasai Y, et al. Baseline and changes in serum uric acid independently predict 11-year incidence of metabolic syndrome among community-dwelling women. J Endocrinol Invest 2018;41(8):959-68.

16. Richette P, Perez-Ruiz F, Doherty M, et al. Improving cardiovascular and renal outcomes in gout: what should we target? Nat Rev Rheumatol 2014;10(11):654-61.

17. Maruhashi T, Hisatome I, Kihara Y, Higashi Y. Hyperuricemia and endothelial function: From molecular background to clinical perspectives. Atherosclerosis 2018;278:226-31.

18. Luis-Rodriguez D, Donate-Correa J, Martin-Nunez E, et al. Serum urate is related to subclinical inflammation in asymptomatic hyperuricaemia. Rheumatology (Oxford) 2021;60(1):371-9.

19. Lu J, Sun M, Wu X, et al. Urate-lowering therapy alleviates atherosclerosis inflammatory response factors and neointimal lesions in a mouse model of induced carotid atherosclerosis. FEBS $\mathrm{J}$ 2019;286(7):1346-59.

20. Jayachandran M, Qu S. Harnessing hyperuricemia to atherosclerosis and understanding its mechanistic dependence. Med Res Rev 2021;41(1):616-29.

21. Bjornstad P, Laffel L, Lynch J, et al. Elevated Serum Uric Acid Is Associated With Greater Risk for Hypertension and Diabetic Kidney Diseases in Obese Adolescents With Type 2 Diabetes: An Observational Analysis From the Treatment Options for Type 2 Diabetes in Adolescents and Youth (TODAY) Study. Diabetes Care 2019;42(6):1120-8.

22. Wei F, Sun N, Cai C, et al. Associations between serum uric acid and the incidence of hypertension: a Chinese senior dynamic cohort study. J Transl Med 2016;14(1):110.

23. Cicero AF, Salvi P, D'Addato S, Rosticci M, Borghi C, Brisighella Heart Study g. Association between serum uric acid, hypertension, vascular stiffness and subclinical atherosclerosis: data from the Brisighella Heart Study. J Hypertens 2014;32(1):57-64.

24. Wu AH, Gladden JD, Ahmed M, Ahmed A, Filippatos G. Relation of serum uric acid to cardiovascular disease. Int J Cardiol 2016;213:4-7.

25. Liu F, Hui S, Hidru TH, et al. The Prevalence, Distribution, and Extent of Subclinical Atherosclerosis and Its Relation With Serum Uric Acid in Hypertension Population. Front Cardiovasc Med 2021;8:638992.

26. Cicero AFG, Fogacci F, Giovannini M, et al. Serum uric acid predicts incident metabolic syndrome in the elderly in an analysis of the Brisighella Heart Study. Sci Rep 2018;8(1):11529.

27. Son M, Seo J, Yang S. Association between dyslipidemia and serum uric acid levels in Korean adults: Korea National Health and Nutrition Examination Survey 2016-2017. PLoS One 
2020;15(2):e0228684.

28. Sekula P, Del Greco MF, Pattaro C, Kottgen A. Mendelian Randomization as an Approach to Assess Causality Using Observational Data. J Am Soc Nephrol 2016;27(11):3253-65.

29. Biradar MI, Chiang KM, Yang HC, Huang YT, Pan WH. The causal role of elevated uric acid and waist circumference on the risk of metabolic syndrome components. Int J Obes (Lond) 2020;44(4):86574.

30. Li L, Song Q, Yang X. Lack of Associations between Elevated Serum Uric Acid and Components of Metabolic Syndrome Such as Hypertension, Dyslipidemia, and T2DM in Overweight and Obese Chinese Adults. J Diabetes Res 2019;2019:3175418.

31. Drivelegka P, Forsblad-d'Elia H, Angeras 0 , et al. Association between serum level of urate and subclinical atherosclerosis: results from the SCAPIS Pilot. Arthritis Res Ther 2020;22(1):37.

\section{Tables}

Table 1 Characteristics of study participants according to uric acid quartiles 


\begin{tabular}{|c|c|c|c|c|c|}
\hline & Q1 & Q2 & Q3 & Q4 & $P$ value \\
\hline & $\mathrm{N}=485(<242)$ & $\mathrm{N}=485(242-293)$ & $\mathrm{N}=489(293-353)$ & $\mathrm{N}=488(\geq 353)$ & \\
\hline ale, n (\%) & $213(43.9)$ & $299(61.6)$ & $384(78.5)$ & $439(90)$ & 0 \\
\hline re, years & $54(47-62)$ & $53(45-60)$ & $50(42-58)$ & $46(35-54)$ & 0 \\
\hline ), years & $5(1-10)$ & $5(0.9-10)$ & $5(2-11)$ & $3(0.3-10)$ & 0.049 \\
\hline ID, n (\%) & $46(9.5)$ & $60(12.4)$ & $40(8.2)$ & $41(8.4)$ & 0.1 \\
\hline ,pertension, $\mathrm{n}$ & $171(35.3)$ & $221(45.6)$ & $206(42.1)$ & $252(51.6)$ & 0 \\
\hline noking, n (\%) & $75(15.5)$ & $124(25.6)$ & $141(28.8)$ & $157(32.2)$ & 0 \\
\hline cohol, n (\%) & $60(12.4)$ & $102(21)$ & $125(25.6)$ & $129(26.4)$ & 0 \\
\hline nI, $\mathrm{kg} / \mathrm{m}^{2}$ & $24(22-26.2)$ & $24.4(22.9-27.7)$ & $26(23.9-28)$ & $26.9(24.7-30)$ & 0 \\
\hline )A1C, \% & $9(7.5-10.6)$ & $8.6(7.3-10.2)$ & $8.5(7.4-10.1)$ & $8.1(6.7-10.4)$ & 0 \\
\hline iG, mmol/L & $7.8(6.2-10.6)$ & $7.8(6.0-10.0)$ & $7.8(6.5-10.3)$ & $7.4(6.0-10.8)$ & 0.199 \\
\hline sulin, $\mu \mathrm{U} / \mathrm{mL}$ & $4.54(2.1-8.1)$ & $4.9(2.5-8.6)$ & $5.5(2.8-11.1)$ & $7.0(3.7-13.8)$ & 0 \\
\hline $3 \mathrm{P}, \mathrm{mg} / \mathrm{L}$ & $0.85(0.49-2.16)$ & $0.96(0.51-2.54)$ & $1.09(0.53-2.43)$ & $1.78(0.86-3.82)$ & 0 \\
\hline $\mathrm{BC}$ & $6(5.1-7.4)$ & $6.1(5.1-7.4)$ & $6.37(5.5-7.6)$ & $6.6(5.6-8.0)$ & 0 \\
\hline 冫R, $\mathrm{mg} / \mathrm{mmoL}$ & $0.67(0.38-1.43)$ & $0.68(0.4-2.19)$ & $0.8(0.34-3.74)$ & $1.29(0.47-5.82)$ & 0 \\
\hline 'eatinine, $\mu \mathrm{mol} / \mathrm{L}$ & $54(47.7-65.5)$ & $61(53-69.7)$ & $67(58-76.4)$ & $71(62-81)$ & 0 \\
\hline $\mathrm{JA}, \mu \mathrm{mol} / \mathrm{L}$ & $206(181-223)$ & $268(256-282)$ & $319(305-337)$ & $410(381-459)$ & 0 \\
\hline $\begin{array}{l}\text { iFR, } \\
/ \mathrm{min} / 1.73^{2}\end{array}$ & $\begin{array}{c}105.7(99.2- \\
115.0)\end{array}$ & $\begin{array}{c}104.3(98.1- \\
113.9)\end{array}$ & $\begin{array}{c}105.3(94.6- \\
114.2)\end{array}$ & $\begin{array}{c}106.1(90.9- \\
118.8)\end{array}$ & 0.418 \\
\hline iP, mmHg & $129(120-143)$ & $131(120-141)$ & $135(126-145)$ & $133(126-143)$ & 0.004 \\
\hline 3P, mmHg & $80(73-87)$ & $81(74-89)$ & $84(79-92)$ & $85(79-92)$ & 0 \\
\hline etS, n (\%) & 277 (57.1) & $353(72.8)$ & $392(80.2)$ & 429 (87.9) & 0 \\
\hline 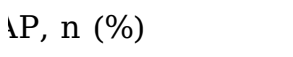 & 275 (56.7) & $258(53.2)$ & $244(49.9)$ & $238(48.8)$ & 0.058 \\
\hline $\mathrm{MT}, \mathrm{mm}$ & $1.3(1.1-1.3)$ & $1.2(1.1-1.3)$ & $1.2(1.1-1.3)$ & $1.2(1.1-1.3)$ & 0.179 \\
\hline$\because, \mathrm{mmol} / \mathrm{L}$ & $4.43(3.8-5.1)$ & $4.34(3.6-5.2)$ & $4.38(3.8-5.1)$ & $4.44(3.8-5.2)$ & 0.012 \\
\hline ;, $\mathrm{mmol} / \mathrm{L}$ & $1.26(0.9-2.0)$ & $1.68(1.1-2.6)$ & $1.88(1.2-3.0)$ & $2.09(1.4-3.9)$ & 0 \\
\hline JL-C, mmol/L & $1.13(0.9-1.4)$ & $1.03(0.9-1.3)$ & $0.99(0.8-1.2)$ & $0.95(0.8-1.2)$ & 0 \\
\hline )L-C, mmol/L & $2.69(2.1-3.3)$ & $2.51(1.9-3.5)$ & $2.61(2.0-3.2)$ & $2.48(1.8-3.1)$ & 0.189 \\
\hline
\end{tabular}

ACR, albumin/creatinine ratio; BMI, body mass index; CIMT, carotid intima-media thickness; CAP, carotid artery plaques; CRP, C-reactive protein; DBP, diastolic blood pressure; DD, duration of diabetes; eGFR, estimated glomerular filtration; FBG, fasting blood glucose; HDL-C, high-density lipoprotein cholesterol; LDL-C, low-density lipoprotein cholesterol; MetS, metabolic syndrome; SBP, systolic blood pressure; SUA, serum uric acid; TC, total cholesterol; TG, total triglycerides; WBC, white blood cells;

\section{Table 2 Correlation between SUA and other parameters}




\begin{tabular}{lcc}
\hline Variable & Correlation coefficient & P value \\
\hline BMI, kg/m ${ }^{2}$ & 0.325 & 0 \\
CRP, mg/L & 0.019 & 0.514 \\
WBC & 0.129 & 0 \\
HbA1C, \% & -0.173 & 0 \\
ACR, mg/mmoL & 0.152 & 0 \\
FBG, mmol/L & -0.044 & 0.056 \\
Creatinine, $\mu \mathrm{mol} / \mathrm{L}$ & 0.292 & 0 \\
eGFR, ml/min/1.732 & -0.33 & 0 \\
TC, mmol/L & 0.105 & 0 \\
TG, mmol/L & 0.184 & 0 \\
HDL-C, mmol/L & -0.148 & 0 \\
LDL-C, mmol/L & 0.013 & 0.56 \\
SBP, mmHg & 0.092 & 0 \\
DBP, mmHg & 0.099 & 0 \\
CIMT, mm & -0.06 & 0.355 \\
\hline
\end{tabular}

All correlation coefficients were calculated after adjustment for age, gender, and diabetes duration.

Table 3 Adjusted ORs and 95\% CIs for MetS and CAP according to SUA quartiles

\begin{tabular}{lccccc}
\hline & Q1 & Q2 & Q3 & Q4 & P value \\
\cline { 2 - 4 } & \multicolumn{5}{c}{ OR (95\% CI) } \\
\cline { 3 - 5 } CAP \\
Model 1 & 1 & $0.85(0.64-1.14)$ & $0.90(0.67-1.21)$ & $1.07(0.78-1.47)$ & $\mathbf{0 . 4 0 8}$ \\
Model 2 & 1 & $0.77(0.58-1.04)$ & $0.82(0.60-1.11)$ & $0.90(0.65-1.25)$ & $\mathbf{0 . 3 3 2}$ \\
Model 3 & 1 & $0.80(0.59-1.08)$ & $0.85(0.62-1.16)$ & $0.97(0.69-1.37)$ & $\mathbf{0 . 3 8}$ \\
Model 4 & 1 & $0.82(0.54-1.24)$ & $0.87(0.56-1.35)$ & $1.07(0.65-1.76)$ & $\mathbf{0 . 5 5 9}$ \\
MetS & & & & & \\
Model 1* & 1 & $2.00(1.52-2.63)$ & $2.94(2.18-3.96)$ & $5.13(3.63-7.25)$ & $\mathbf{0}$ \\
Model 2* & 1 & $1.96(1.49-2.58)$ & $2.94(2.18-3.97)$ & $5.17(3.65-7.32)$ & $\mathbf{0}$ \\
Model 3* & 1 & $1.41(1.01-1.97)$ & $1.69(1.18-2.42)$ & $2.66(1.77-4.01)$ & $\mathbf{0}$ \\
Model 4* & 1 & $2.01(1.24-3.25)$ & $1.99(1.17-3.37)$ & $2.91(1.54-5.51)$ & $\mathbf{0 . 0 0 3}$
\end{tabular}

Model 1 adjusted for age and gender.

Model 2 further adjusted for alcohol drinking, smoking, duration of diabetes, hypertension, and history of CVD. 
Model 3 further adjusted for BMI.

Model 4 further adjusted for eGFR, CRP, FBG, HbA1C, TG, TC, HDL-C, and LDL-C.

Model $1 *$ adjusted for age and gender.

Model 2* further adjusted for alcohol drinking, smoking, duration of diabetes, and history of CVD.

Model 3* further adjusted for BMI.

Model 4* further adjusted for eGFR, CRP, FBG, HbA1C, TC, and LDL-C.

Table 4 Association of SUA quartiles with MetS components

\begin{tabular}{lccccc}
\hline & Q1 & Q2 & Q3 & Q4 & P value \\
\cline { 2 - 6 } & \multicolumn{7}{c}{ OR (95\% CI) } \\
\hline Overweight/Obesity & 1 & $1.56(1.08-2.23)$ & $2.56(1.74-3.76)$ & $3.45(2.25-5.28)$ & 0 \\
Hypertension & 1 & $1.40(0.94-2.08)$ & $1.41(0.93-2.15)$ & $2.13(1.34-3.38)$ & 0.015 \\
Dyslipidemia (TG) & 1 & $1.61(1.09-2.37)$ & $2.56(1.70-3.85)$ & $2.71(1.73-4.25)$ & 0 \\
Dyslipidemia (TC) & 1 & $0.71(0.32-1.57)$ & $1.41(0.68-2.94)$ & $2.51(1.17-5.38)$ & 0.013 \\
Dyslipidemia (HDL-C) & 1 & $1.45(0.99-2.12)$ & $1.36(0.91-2.03)$ & $1.52(0.98-2.37)$ & 0.195
\end{tabular}

Adjusted for age, sex, alcohol drinking, smoking, duration of diabetes, self-reported CVD, BMI, CRP, HbA1C, FBG, eGFR.

\section{Figures}
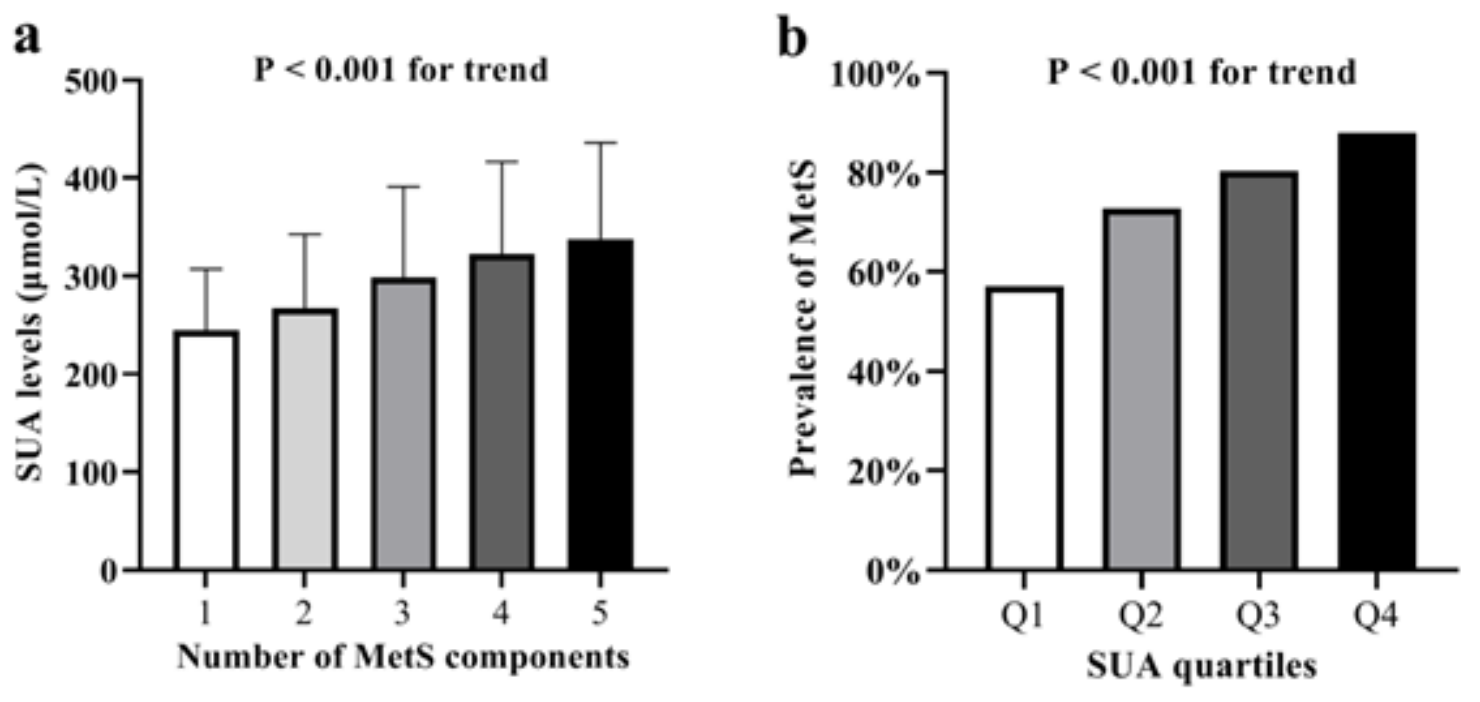

Figure 1

(a) Serum uric acid levels according to the number of MetS components. (b) Comparison of the prevalence of MetS among the four SUA quartile groups. 